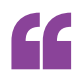

the deposition

of insulin

mirrored the

spread of $\mathrm{p}$-tau

in the brain

ALZHEIMER DISEASE

\title{
Is p-tau the missing link between insulin resistance and AD?
}

Defects in insulin signalling have been implicated in the pathogenesis of Alzheimer disease (AD), but the underlying mechanisms have remained obscure. According to new research reported in Brain, hyperphosphorylated tau protein ( $\mathrm{p}$-tau), one of the hallmarks of $\mathrm{AD}$, promotes the oligomerization and accumulation of insulin in brain neurons, thereby potentially contributing to insulin resistance.

"Diabetes mellitus is a well-known risk factor for $\mathrm{AD}$, and early events in $\mathrm{AD}$ pathogenesis include insulin resistance and alterations in glucose metabolism," explains first author Patricia Rodriguez. "When we reviewed the literature on this topic, we found that the question of how insulin is distributed in the brain in $\mathrm{AD}$ and other tauopathies had been widely neglected."

Rodriguez and colleagues used immunohistochemistry to compare insulin distribution patterns in brain tissue from individuals with and without $\mathrm{AD}$. Insulin levels were found to be elevated in the brains of the patients with $\mathrm{AD}$, particularly in the hippocampus. Moreover, the deposition of insulin mirrored the spread of p-tau in the brain, although no evidence of a direct physical interaction between the two proteins was found.

To further explore the relationship between $\mathrm{p}$-tau and insulin, the researchers switched their attention to cellular models of tau hyperphosphorylation. Treatment of rat cortical neurons with the phosphatase inhibitor okadaic acid, which is known to induce hyperphosphorylation of tau, resulted in oligomerization and accumulation of insulin. However, silencing of tau via transfection with small interfering RNA inhibited insulin accumulation in these cells. Experiments in human neuroblastoma cells that were engineered to overexpress tau provided additional evidence that intraneuronal accumulation of insulin depends on tau hyperphosphorylation.

"We have shown for the first time that endogenous insulin undergoes oligomerization in the brain, although the exact mechanism by which tau hyperphosphorylation promotes insulin uptake and accumulation remains unclear," concludes senior author Angel Cedazo-Minguez. "Given the relevance of insulin for glucose uptake and metabolic regulation, as well as its close relationship with neuronal plasticity and memory formation, we consider that this novel pathological mechanism could open up a completely new approach for understanding neurodegeneration in $\mathrm{AD}$, and for the development of new therapeutic strategies against this disease."

Heather Wood

ORIGINAL ARTICLE Rodriguez-Rodriguez, P. et al. Tau hyperphosphorylation induces oligomeric insulin accumulation and insulin resistance in neurons. Brain http://dx.doi.org/10.1093/brain/ awx256 (2017) 